\title{
La actividad física como elemento de participación y calidad de vida en las personas mayores
}

\section{Physical activity as an element of participation and quality of life among elderly people}

\author{
Inmaculada Herranz Aguayo, Juan Lirio Castro, Esther Portal Martínez y Enrique Arias Fernández
}

Universidad de Castilla La Mancha, España

Disponible online 31 de agosto de 2013

\begin{abstract}
El presente trabajo relaciona la participación a través de la actividad física como elemento que contribuye a la salud y la calidad de vida de las personas mayores. Así desarrolla un análisis de tipo descriptivo con datos secundarios que pretende explorar la posición del colectivo de personas mayores en relación a la actividad física, su incidencia y características con el objetivo de indagar su mayor o menor importancia en relación a la calidad de vida en este grupo de edad.

Las categorías de análisis que articulan los resultados se agrupan en: estructura de la población mayor por sexo y edad, calidad de vida y salud percibida, estilos de vida y actividades cotidianas y principales características de la actividad física en personas mayores. Para aumentar la capacidad comparativa y de análisis se ha incluido la totalidad de tramos de edad en cada una de las variables. Entre los resultados se destaca el buen estado de salud y la alta participación en actividades física de las personas mayores en comparación con otros grupos de edad.
\end{abstract}

Palabras clave: Actividad Física, Participación; Calidad de Vida; Personas Mayores.

This study investigated participation in physical activity as an element that improves health and quality of life among elderly people. We present a descriptive analysis using secondary data to explore their attitudes toward physical activity, its frequency, and characteristics.

The following categories were analysed: population structure by sex and age; quality of life and perceived health; lifestyles and daily activities; and the characteristics of physical activities among elderly people. To increase the comparative and analytical power of the study, all age ranges were included in each variable. The results highlight the good state of health and high participation in physical activity among elderly people compared to other age groups.

Key words: Physical Activity; Participation; Quality of Life; Elderly People.

Correspondencia: Inmaculada Herranz Aguayo, Universidad de Castilla La Mancha, Facultad de Ciencias Sociales, Avenida Real Fábrica de Seda s/n, 45600 Talavera de la Reina - Toledo. E-mail: Inmaculada.Herranz@uclm.es; E-mail de los otros autores: Juan Lirio Castro: Juan.Lirio@uclm. es, Esther Portal Martínez: Esther.Portal@uclm.es, Enrique Arias Fernández: Enrique.AFernandez@uclm.es 
En general la salud entendida en sentido amplio ha sido considerada como uno de los componentes esenciales en la calidad de vida. Así lo manifestaron por ejemplo Cummins y Cahill (2000) cuando mencionan siete ámbitos que se repiten en todos los estudios desarrollados sobre calidad de vida, a saber: salud, intimidad, bienestar material, productividad, bienestar emocional, seguridad y comunidad. Del mismo modo, en nuestro contexto, un estudio desarrollado por Fernández-Ballesteros y Maciá (1993) situó la salud como componente principal en la calidad de vida. La OMS (2002) resalta que la salud es fundamental en la calidad de vida de las personas mayores al definir el envejecimiento activo como el proceso de optimización de oportunidades de salud, participación y seguridad para mejorar la calidad de vida.

La actividad y la participación tienen efectos positivos tanto en la salud como en la calidad de vida (Alonso, Lirio y Herranz, 2007; Monchietti y Krzemien, 2000; Yuni y Urbano, 2007) así como en el proceso de transición desde la vida laboral a la jubilación y en el desarrollo personal e integración social (Moen, Fields, Quick y Hofmeister, 2000). Participar en consecuencia se entiende como la realización de actividades que permiten a las personas mayores su desarrollo e integración personal a la vez que mantener su calidad de vida. Monchietti y Krzemien (2000) incluyen un matiz interesante cuando al hablar de participación social se refieren a la "participación social significativa", proceso de interacción personal que consiste en tomar parte en forma activa y comprometida en una actividad conjunta, la cual es percibida por la persona como beneficiosa. Para ellos la participación social haría referencia a sistemas de apoyo o suministros psicosociales como uniones entre individuos, caracterizados por: a) ayuda material; b) asistencia física; c) compartir pensamientos, sentimientos y experiencias, y d) contactos sociales positivos. Finalmente, consideran que la participación social significativa parece hallarse en básicamente cuatro áreas: la educativa, la ocupación laboral, la actividad física y recreativa de carácter grupal, y las relaciones sociales propiamente dichas donde el fin es la interacción mutua y la integración social.

Diferentes autores han estudiado la participación social de las personas mayores en relación a diferentes tipos de actividades. Vega, Buz y Bueno (2002) en un estudio sobre la participación social, desarrollado con 501 personas mayores de 60 años de la ciudad de Salamanca concluyen que las actividades que hacen a diario muestran que la participación social de los mayores es baja; además estos tienen preferencia por actividades de tipo individual, es decir, que no implican relación social. En general, las frecuencias más altas se registran en actividades de ocio que no exigen esfuerzo intelectual o conciencia social. En cuanto a las variables mediadoras en las actividades y la participación social de los mayores, los autores señalan las siguientes: 1) red de apoyo social, referida tanto a la frecuencia, intensidad y duración de las relaciones entre los diferentes individuos que conforman dicha red; 2) salud y autonomía funcional; y 3) heteropercepción y autopercepción de los mayores.
Erlinghagen y Hank (2005) presentan las conclusiones de un estudio realizado en diez países europeos (Suecia, Dinamarca, Alemania, Países Bajos, Francia, Suiza Austria, Italia, España y Grecia) mediante una encuesta a 22.000 ciudadanos mayores de 50 años, resultado de la explotación de la encuesta SHARE (Salud, Envejecimiento y Jubilación en Europa), siendo uno de los múltiples análisis la participación de las personas mayores en actividades voluntarias. España aparece con el menor índice de colaboración voluntaria de todos los países estudiados, a gran distancia de Europa del norte y central. El estudio refiere un amplio conjunto de covariables: actividades sociales tales como la ayuda o la asistencia no profesional y la participación en las actividades de una organización (un club deportivo, una iglesia, o un partido político, por ejemplo); sexo, edad y estado civil; nivel educativo y, salud actual percibida. Con respecto al trabajo de voluntariado y su relación con la salud, encontramos índices de actividad mucho más bajos entre quienes perciben su estado de salud actual como normal o malo, en relación a los que declaran un estado de salud bueno o mejor. Como puede comprobarse, la salud aparece en casi todos los estudios como una variable mediadora o que condiciona de forma importante las posibilidades de participación de las personas mayores, influyendo decisivamente en la calidad de vida. Por ello participar resulta esencial para las personas mayores si bien se entiende que existen diferentes tipos y niveles de participación. En esta dirección apunta Peréz (2003) cuando al definir la participación además de la idea de formar parte añade el matiz de sentirse actores. Lirio, Alonso y Herranz (2009) afirman que la participación social no hay que entenderla como un proceso de todo o nada, sino que más bien se desarrolla en niveles. Es decir, como una herramienta de empoderamiento que las personas pueden utilizar en función de sus realidades y necesidades, y a diferentes niveles de intensidad. Tomar la decisión de participar en una actividad y mantener cierto grado de implicación en ella depende de variables personales y también de las características del entorno, como el ambiente físico, las características del grupo con el que se desarrolla o desarrollaría la actividad, el clima de seguridad psicológica que se percibe, etc. (Csikszentmihalyi, 1998; Omoto y Snyder, 1995).

A pesar de ser un proceso, y no una posición finalista de todo o nada, no se puede dejar de reconocer que participar permite la consecución de objetivos concretos, y proporciona un sentimiento de competencia que refuerza la autoconfianza y motiva para seguir participando en dichas actividades y así permanecer "activo". Como ya determinara Csikszentmihalyi (1998), las personas experimentan satisfacción cuando están completamente involucradas en la realización de una actividad desafiante, y esto les hace desear mantenerse adheridas a su práctica. Así entendida, y asumiendo la diversidad del colectivo de personas mayores, no debemos ignorar que la vivencia singular de la actividad da lugar a diferentes "itinerarios" o estilos de participación a lo largo del tiempo (Martínez et al, 2007). 
Desde esta perspectiva, la participación se convierte en un elemento que contribuye a la salud y calidad de vida de las personas mayores. Por ello, es interesante analizar el nivel de participación en actividades físicas por parte de las personas mayores dada la importancia que la misma puede tener en la prevención y mantenimiento de la salud al ser una más de las posibles actividades en que pueden participar en nuestro entorno.

\section{Método}

La metodología que se va a emplear para el desarrollo y fundamentación de este artículo se centra en un análisis descriptivo con datos secundarios ofrecidos por las principales entidades y fuentes estadísticas de producción de datos cuantitativos en esta materia:

- Cifras Oficiales de Población. Explotación Estadística del Padrón a 1 de Enero de 2012. Instituto Nacional de Estadística. 2013.

- Encuesta Nacional de Salud 2006. Instituto Nacional de Estadística y Ministerio de Sanidad, Política Social e Igualdad. 2008.

- Encuesta de Empleo del Tiempo 2009-2010. Instituto Nacional de Estadística. 2011.

- Informe 2010. Las Personas Mayores en España. Datos estadísticos nacionales. Observatorio de Personas Mayores del Imserso. Ministerio de Sanidad, Política Social e Igualdad.

La población objeto de estudio son las personas mayores de 65 años en tanto que suponen un consenso generalizado como tramo de edad representativo del colectivo "personas mayores". A pesar de ser las "personas mayores" la población objeto de estudio, se ha incorporado la variable edad en la totalidad de categorías y variables analizadas para mejorar la capacidad comparativa y analítica. El ámbito de investigación se corresponde con los resultados a nivel nacional, sin descomponerlos por Comunidades Autónomas.

Dado que el objetivo de este trabajo se fundamenta en la valoración de la actividad física como parte de la participación social para mejorar la calidad de vida de las personas mayores, se ha articulado el análisis y resultados en relación a las siguientes categorías de análisis:

1. Estructura de la población mayor por sexo y edad.

2. Calidad de vida y salud percibida:

2.2. Esperanza de vida al nacer y a los 65 años.

2.3. Percepción del estado de salud.

3. Estilos de vida y actividades realizadas:

3.1. Actividades realizadas a lo largo del día por edad en porcentaje de personas.

3.2. Tiempo dedicado a actividades a lo largo del día. Duración media diaria (DMD horas).

4. Actividad física:

4.1. Ocio dinámico.

4.2. Realización de actividad física por edad.

4.3. Intensidad de la actividad física por edad.

4.4. Horas dedicadas a la actividad física por edad.

\section{Resultados}

Como se ha comentado, la actividad física puede constituirse en una forma de participación que mejora la calidad de vida. Los principales elementos de la participación incluirían: las relaciones sociales, la participación en entidades y voluntariado, la participación en el sistema productivo, el ocio- dinámico y, dentro de él, la actividad física. Este trabajo se ha centrado en uno de sus elementos, la actividad física. Lo que se presenta a continuación es un análisis descriptivo de los principales elementos estructurales de la población mayor actual en España y su posición predominante ante la actividad física. Tal y como aparece en la metodología el análisis se articula en torno a tres grandes bloques. En primer lugar, se presentan las características socio-demográficas básicas sobre la población mayor y la calidad de vida. En segundo lugar, algunos datos sobre la vida cotidiana y estilo de vida con el objetivo de ubicar la actividad física entre las actividades diarias de la población mayor. Para terminar, se incluye un acercamiento a las principales características de la actividad física en las personas mayores de 65 años.

\section{Estructura de la población mayor por sexo y edad}

Las características básicas de estructura sociodemográfica de la población sirven para detectar las poblaciones susceptibles de participar en actividades físicas y las características de las mismas. En el caso de las personas mayores las representaciones sociales sobre la vejez han actuado como limitantes en el desarrollo de la actividad física en personas mayores al presuponerse una visión deficitaria del proceso de envejecimiento. De alguna manera, los datos socio-demográficos que se presentan tienen el objetivo de matizar e incluso cuestionar estas representaciones sociales.

Respecto a la distribución de la población mayor por sexo y edad cabe resaltar que el $69.6 \%$ de la población mayor de 65 años se encuentra entre los 65-79 años como se observa en la Figura 1. De alguna manera podría afirmarse que aproximadamente el $70 \%$ de la población mayor se corresponde con las edades más jóvenes dentro de sus tramos de edad.

\section{Figura 1}

Distribución de la población mayor de 65 años por sexo y edad, 2012

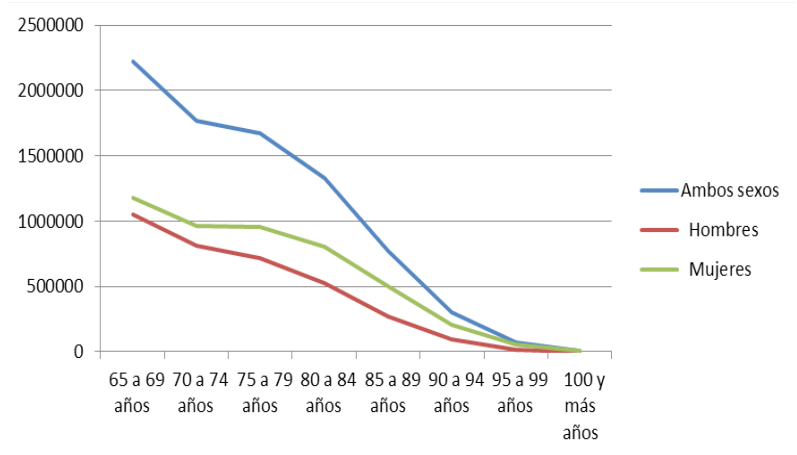

Nota: Elaboración propia a partir de datos de la Explotación estadística del Padrón. 1 de enero 2012, INE. 
En relación con las diferencias por sexo, el gráfico presentado muestra un mayor nivel de supervivencia de las mujeres frente a los hombres que genera, como consecuencia, la feminización de la población mayor. Este mayor porcentaje de mujeres se justifica en la desigual distribución por sexo de la esperanza de vida.

Uno de los mejores indicadores de salud y de calidad de vida, además del nivel social, económico y sanitario de un país, es la Esperanza de vida. Este dato como se observa en la Tabla 1 nos indica el excelente estado de salud de la población española y en concreto los mayores. España se sitúa en uno de los primeros puestos de Europa en este índice. La principal causa es el descenso tanto de la mortalidad infantil como de la mortalidad de la población entre 70 y 80 años. Los datos señalan que existe una importante diferencia entre sexos, siendo las mujeres las que más años viven. No obstante en España, a la edad de 65 años, se prevé que los hombres vivan 18.5 años más, mientras que las mujeres vivirían 22.4 años más, casi una cuarta parte de la vida.

Las políticas deben tener en cuenta la continuidad de la vida y no compartimentar tanto las edades, asignando, por ejemplo, el aprendizaje y la educación a la infancia y juventud, el trabajo a la edad adulta y el ocio a la vejez. Un mayor período de vejez debe motivar para cambiar las edades y las formas de jubilación, de participación social, de aprendizaje y de actividad, y no condicionar estas cualidades a períodos cerrados y anteriores del curso de vida (IMSERSO, 2012).

Tabla 1

Esperanza de vida al nacimiento y esperanza de vida a los 65 años

\begin{tabular}{|c|c|c|c|c|c|c|}
\hline & \multicolumn{3}{|c|}{ Esperanza de vida al nacimiento } & \multicolumn{3}{|c|}{$\begin{array}{l}\text { Esperanza de vida a los } \\
65 \text { años }\end{array}$} \\
\hline & $\begin{array}{l}\text { Ambos } \\
\text { sexos }\end{array}$ & Hombres & Mujeres & $\begin{array}{l}\text { Ambos } \\
\text { sexos }\end{array}$ & Hombres & Mujeres \\
\hline \multicolumn{7}{|l|}{$\begin{array}{c}\text { Total } \\
\text { Nacional }\end{array}$} \\
\hline 2011 & 82.1 & 79.2 & 85.0 & 20.5 & 18.5 & 22.4 \\
\hline 2010 & 82.0 & 78.9 & 84.9 & 20.4 & 18.3 & 22.3 \\
\hline 2009 & 81.6 & 78.5 & 84.6 & 20.2 & 18.1 & 22.0 \\
\hline 2008 & 81.2 & 78.2 & 84.3 & 20.0 & 18.0 & 21.8 \\
\hline 2007 & 80.9 & 77.8 & 84.1 & 19.8 & 17.7 & 21.7 \\
\hline 2006 & 80.9 & 77.7 & 84.2 & 19.9 & 17.8 & 21.7 \\
\hline 2005 & 80.3 & 77.0 & 83.5 & 19.3 & 17.2 & 21.1 \\
\hline 2004 & 80.3 & 77.0 & 83.6 & 19.4 & 17.3 & 21.3 \\
\hline 2003 & 79.7 & 76.4 & 83.0 & 18.9 & 16.8 & 20.8 \\
\hline 2002 & 79.8 & 76.4 & 83.1 & 19.0 & 16.9 & 20.9 \\
\hline 2001 & 79.7 & 76.3 & 83.1 & 19.0 & 16.9 & 20.8 \\
\hline 2000 & 79.3 & 75.9 & 82.7 & 18.8 & 16.7 & 20.6 \\
\hline 1999 & 78.8 & 75.4 & 82.3 & 18.3 & 16.2 & 20.2 \\
\hline 1998 & 78.8 & 75.4 & 82.3 & 18.3 & 16.2 & 20.2 \\
\hline 1997 & 78.7 & 75.2 & 82.2 & 18.4 & 16.3 & 20.2 \\
\hline 1996 & 78.2 & 74.6 & 81.8 & 18.3 & 16.2 & 20.1 \\
\hline 1995 & 78.1 & 74.5 & 81.7 & 18.2 & 16.1 & 20.0 \\
\hline 1994 & 78.0 & 74.5 & 81.6 & 18.2 & 16.1 & 19.9 \\
\hline 1993 & 77.7 & 74.1 & 81.2 & 17.9 & 15.9 & 19.6 \\
\hline 1992 & 77.5 & 73.9 & 81.2 & 17.9 & 15.9 & 19.6 \\
\hline 1991 & 77.1 & 73.5 & 80.7 & 17.6 & 15.6 & 19.2 \\
\hline
\end{tabular}

Nota: Indicadores demográficos básicos. Serie 1991-2011, INE.
Además de la esperanza de vida, el concepto subjetivo de salud, es muy empleado en los estudios de calidad de vida porque, recoge de algún modo, la valoración del estado de salud de los individuos de manera general (refleja el estado físico, las enfermedades que se han padecido, ...) pero también, y es lo más importante, refleja los factores sociales, económicos y del entorno de la persona (IMSERSO, 2012). Este indicador predice futuras situaciones, necesidades, habilidades funcionales de la población e indica el estado de salud general, predice necesidades asistenciales y sirve para la organización de programas. La sensación de bienestar se relaciona con las acciones y actividades que realiza el individuo. De ahí que este dato indique el impacto de las políticas sanitarias y sociales.

En cuanto a la situación actual, los jóvenes son los que mejor percepción tienen de su salud, considerándola buena y muy buena, y va decreciendo a medida que aumenta la edad. Por el contrario, los porcentajes de una percepción de salud mala o muy mala, aumentan a medida que lo hace la edad, aunque con porcentajes muy bajos. En general, la percepción del estado de salud de los españoles se sitúa entre bueno y muy bueno, de hecho el 40.5\% abarca ambos ítems (IMSERSO, 2012).

Como en la mayoría de las variables tratadas, la diferenciación por género hace acto de presencia también en cuanto a la percepción del estado de salud. En este caso son los hombres mayores los que se perciben con mejor salud que las mujeres mayores, un $48.6 \%$ frente a un $34.4 \%$. Sin embargo un análisis más atento a los tramos de edad desvela que en el caso de los mayores de $65-74$ tan solo el $16.5 \%$ considera su salud "mala o muy mala" frente al $46.9 \%$ que la considera "buena o muy buena" ( el porcentaje no explicado se encuentra en el "regular") (IMSERSO, 2012).

Esta percepción de la salud de los mayores está influida por sus niveles económicos y formativos. Los datos de IMSERSO revelan que los mayores de 65 años con un nivel académico alto poseen mejores perspectivas de salud que aquellos con niveles académicos inferiores. Es necesario apuntar que la variable nivel académico está estrechamente relacionada con el nivel económico, por lo que se puede concluir que la percepción sobre el estado de salud de los mayores depende significativamente del nivel socioeconómico de los mismos.

\section{Vida cotidiana y estilo de vida}

No es propósito de este artículo un análisis exhaustivo del estilo de vida de las personas mayores, sino ubicar en sus actividades diarias el peso y posición de la actividad física en su vida cotidiana. Para ello se han seleccionado dos indicadores que pueden orientar en nuestro propósito: la distribución de actividades por grupos de edad en porcentaje de personas y el tiempo dedicado a cada una de ellas.

Si se consideran las actividades más importantes en la vida cotidiana de los distintos tramos de edad, las actividades deportivas no son de las más relevantes como se puede observar en la Figura 2. Entre las principales actividades en todos los tramos 
de edad se encuentran los cuidados personales, medios de comunicación, tiempo empleado en trayectos y tiempo no identificado y vida social y diversión. Algunas actividades se elevan marcadamente en los tramos de edad más propios de la actividad, así el trabajo remunerado se eleva en las edades activas y se hace menor en las más jóvenes y en los mayores de 65 años. Sin embargo, si se realiza el análisis por actividades, resalta entre otras la diferencia en porcentaje de personas que hacen actividades deportivas por tramo de edad. Siendo nuestro objetivo principal cabe resaltar que, de todos los tramos de edad, los mayores de 65 años son los que representan en mayor porcentaje de personas en actividades deportivas. Ni siquiera el tramo de edad más joven (menos de 25 años) supera a las personas mayores.

\section{Figura 2.}

Actividades realizadas en el día. Tantos por ciento de la población.

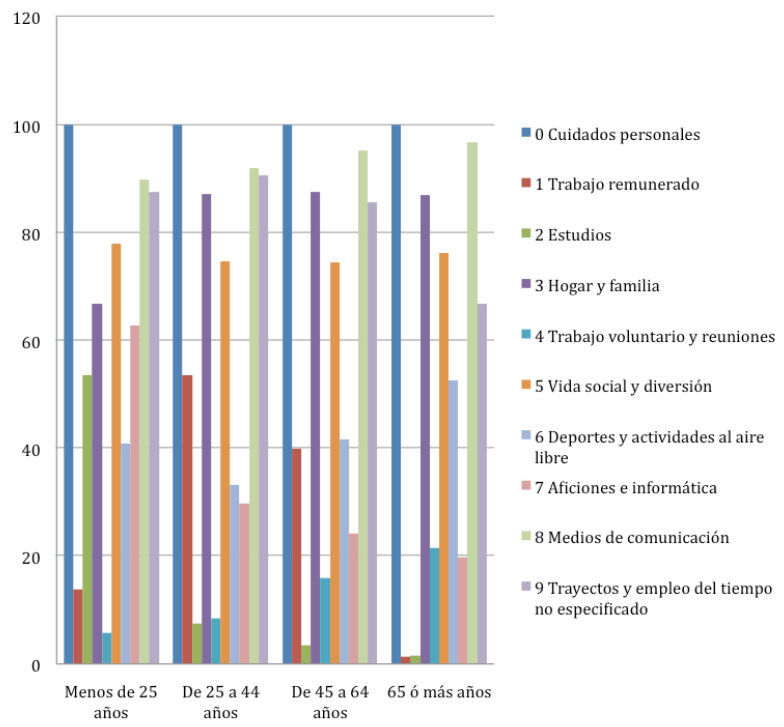

Nota: Elaboración propia a partir de los datos de la Encuesta de Empleo del Tiempo, 2006, INE.

Un nivel de avance más en la misma línea de análisis lo constituye el tiempo diario dedicado a esta distribución de actividades (Figura 3). Cabría pensar que aunque haya más personas mayores vinculadas a actividades deportivas el tiempo dedicado no tiene por qué ser superior. En esta dirección, la Encuesta de Empleo del Tiempo 2009-2010 (INE, 2011) vuelve a reiterar el papel fundamental de esta actividad en la vida de los mayores en relación al resto de intervalos de edad. Son los jóvenes menores de 25 años y los mayores de 65 años los que dedican mayor tiempo al día a estas actividades, un total de 2 horas/día. En el caso de los tramos de edad en edades intermedias la dedicación baja a 1 hora/día. La diferencia debe ser explicada teniendo en cuenta la incidencia en las edades intermedias de actividades como el hogar y los hijos o el trabajo remunerado. En definitiva, se puede concluir que la actividad deportiva y la dedicación horaria a la misma es importante en la vida de los mayores.
Figura 3

Duración media diaria en horas dedicada a distintas actividades por tramos de edad.

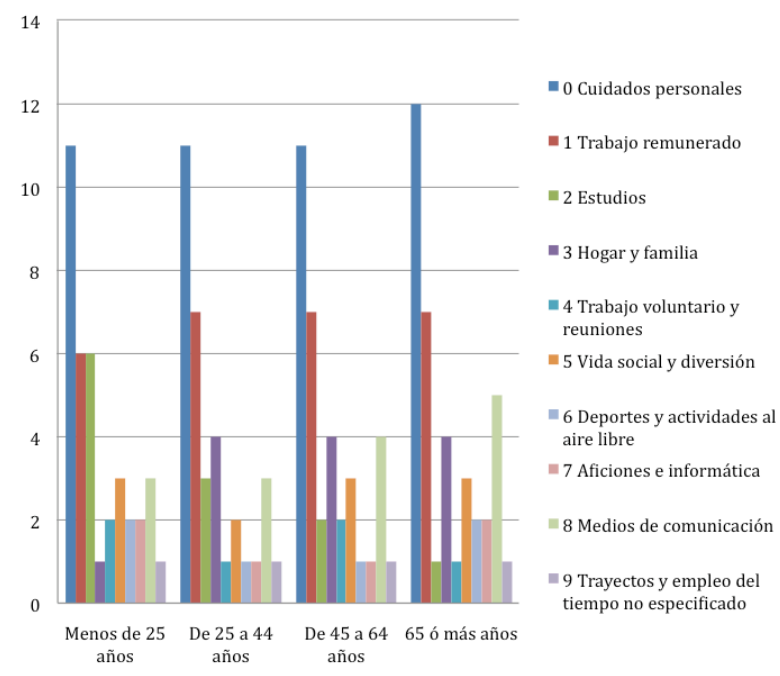

Nota: Elaboración propia a partir de los datos de la Encuesta de Empleo del Tiempo 2006, INE.

\section{Actividad física}

Dado que la actividad física supone una de las actividades relevantes en la participación y vida cotidiana de la población mayor, a continuación se describirán algunas características de esta actividad.

El Informe 2010 sobre Personas Mayores (IMSERSO, 2012) conceptualiza las actividad física y deportiva como Ocio - Dinámico incluyendo en esta catalogación: parque/pasear, actividad física y deportiva y bailar. De los mayores de 65 años son los hombres los que más realizan actividad física y deportiva, el $38.5 \%$ de éstos, frente a las mujeres con un $21.2 \%$. Sin embargo estos porcentajes no son homogéneos por tramos de edad dentro de las personas mayores. Entre los mayores de 65 años, en los tramos de edad más jóvenes se eleva el porcentaje, mientras que al elevarse la edad el porcentaje de personas que desarrollan actividad física- deportiva va descendiendo. El tramo de edad de los 65-69 años es el más proclive a la actividad deportiva o actividad física, el $58 \%$ de los mayores en estas edades realizan algún tipo de actividad deportiva o actividad física. A medida que aumenta la edad este porcentaje va disminuyendo hasta ser prácticamente inexistente a partir de los 80 años y más.

Otra fuente estadística relevante lo constituyen los datos aportados por la Encuesta Nacional de Salud 2006 (INE, 2008). En esta ocasión los datos hacen referencia a la conceptualización directa de "ejercicio físico" en tiempo libre en población mayor de 16 años (ver Figura 4). Se trata de una distribución de la población total por tramo de edad en porcentajes de "realización" o "no realización" de ejercicio físico en tiempo libre. 


\section{Figura 4}

Realización de ejercicio físico en el tiempo libre en población adulta por grupo de edad. Tanto por ciento de la población

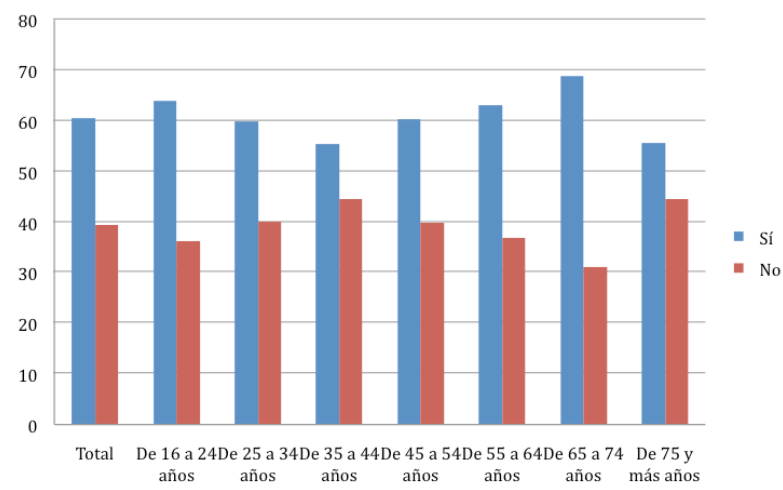

Nota: Elaboración propia a partir de los datos de la Encuesta Nacional de Salud 2006, INE.

Si se considera la totalidad de población, el $60 \%$ de la población mayor de 16 años realiza algún tipo de ejercicio físico. La distribución por edades presenta una representación gráfica similar a una Campana de Gauss invertida, esto es, más pronunciado en el primer intervalo de edad (de 16 a 24 años), descendiendo progresivamente en las edades intermedias (de 25 a 54 años) para posteriormente irse recuperando hasta su máxima representación en el tramo de edad de 65 a 74 años. Más allá de la distribución general, se debe resaltar que el tramo de edad con mayor porcentaje de personas que realizan algún tipo de ejercicio físico en su tiempo libre es el de 65 a 74 años con un $68 \%$ de la población.

Respecto a la intensidad del ejercicio que realizan, el $40 \%$ de los mayores dicen hacer un ejercicio físico ligero, mientras que el $31.1 \%$ realiza una actividad moderada. Respecto a la diferencia por sexo, las mujeres suelen ser menos activas entre los 16 y 34 años y también a partir de los 65, mientras que los hombres acumulan su nivel de inactividad entre los 35 y 64 años. En todos los tramos de edad son los hombres los que realizan una actividad intensa por encima de las mujeres, mientras que las mujeres sobresalen en el nivel de intensidad moderado (IMSERSO, 2012).

Para finalizar se hará referencia a los datos aportados por la Encuesta de Empleo del Tiempo 2009-2010 (INE, 2011) respecto a las personas que realizan "deportes y actividades al aire libre" por sexo y edad y el tiempo dedicado a las mismas (Tabla 2 ). Tal y como se ha anticipado en la comparativa de actividades de la vida cotidiana los mayores son los que más realizan este tipo de actividades, siendo el 52\% de los mayores de 65 años mientras los menores de 25 tan solo realizan estas actividades el 39.6\%. En el tiempo dedicado también se produce esta diferencia siendo las personas mayores de 65 las que más tiempo dedican diariamente a esta actividad. Sin embargo, la distribución por sexo desvela una importante diferencia entre hombres y mujeres. En todas las edades son mayoría los hombres que realizan "deporte y actividades físicas" y les dedican más tiempo. Esta diferencia se mantiene en todos los tramos de edad, pero se hace más marcada en personas mayores llegando a pasar del $61,2 \%$ de los hombres mayores de 65 años al 45,2\% de las mujeres del mismo tramo de edad. Respecto al tiempo dedicado a estas actividades también se mantiene la diferencia llegando a ser de casi 20 minutos entre hombres y mujeres.

\section{Discusión}

El principal eje de discusión se refiere al buen estado de salud y la alta participación en actividad física de las personas mayores en comparación con otros grupos edad. Siendo característica de este tipo de actividad que la intensidad con la que desarrollan la misma es ligera y moderada, frente a otros grupos de edad que realizan actividades físicas de mayor intensidad. Sin embargo, se observa un mayor porcentaje de personas dedicadas a esta actividad así como una mayor dedicación de tiempo a la misma que en el resto de grupos. Este aspecto entraría en contradicción con las representaciones sociales que tienen el resto de edades sobre la vejez y el envejecimiento, que suelen mostrar la dimensión física de este fenómeno como la más deficitaria en el último tramo de la vida (Herranz, Lirio y Portal, 2012; Marazza et al, 2003) y que no parece corroborarse a la luz de los datos estructurales de la población española. $\mathrm{Si}$ a esto sumamos que nos encontramos con que un $70 \%$ de la población mayor española se encuentran en el intervalo de edad de 65-79 años, donde la aparición de enfermedades crónicas y limitantes a nivel físico tienen una menor incidencia, se refuerza aún en mayor medida el buen estado de salud que los mayores presentan y por tanto la elevada posibilidad de implicación e integración en el medio. Por ello consideramos la actividad física como elemento clave para generar programas

Tabla 2

Participación en deporte y actividad física en el tiempo libre por edad y sexo. Tanto por ciento de personas y duración media diaria en horas y minutos.

\begin{tabular}{|c|c|c|c|c|c|c|c|c|c|}
\hline \multirow[t]{2}{*}{ Rangos de edad } & \multicolumn{3}{|c|}{ Ambos sexos } & \multicolumn{3}{|c|}{ Varones } & \multicolumn{3}{|c|}{ Mujeres } \\
\hline & $\begin{array}{c}\text { Personas } \\
\%\end{array}$ & $\begin{array}{l}\text { DMD } \\
\text { horas }\end{array}$ & $\begin{array}{l}\text { DMD } \\
\text { minutos }\end{array}$ & $\begin{array}{c}\text { Personas } \\
\%\end{array}$ & $\begin{array}{l}\text { DMD } \\
\text { horas }\end{array}$ & $\begin{array}{l}\text { DMD } \\
\text { minutos }\end{array}$ & $\begin{array}{c}\text { Personas } \\
\%\end{array}$ & $\begin{array}{l}\text { DMD } \\
\text { horas }\end{array}$ & $\begin{array}{c}\text { DMD } \\
\text { minutos }\end{array}$ \\
\hline Menos de 25 años & 39.6 & 1 & 58 & 42.7 & 2 & 2 & 36.2 & 1 & 53 \\
\hline De 25 a 44 años & 31.8 & 1 & 41 & 33.7 & 1 & 48 & 29.8 & 1 & 32 \\
\hline De 45 a 64 años & 40.9 & 1 & 48 & 42.4 & 1 & 59 & 39.3 & 1 & 37 \\
\hline 65 ó más años & 52 & 2 & 0 & 61.2 & 2 & 17 & 45.2 & 1 & 42 \\
\hline
\end{tabular}

Nota: Fuente: Encuesta de Empleo del Tiempo 2009-2010 (INE)

DMD: Duración media diaria. 
y proyectos que canalicen esta participación, contribuyendo al desarrollo y mantenimiento de altas cotas de salud en el segmento de edad más joven y mayoritaria de la población mayor. Lejos de parecer una actividad vinculada a la juventud, este trabajo pone de manifiesto que nuestros mayores realizan actividad física en mayor medida que otros grupos etarios.

\section{Referencias}

1. Alonso, D, Lirio, J. y Herranz, I. (2007). La participación en la Universidad de Mayores: Beneficios en la calidad de vida. En D. Alonso, J. Lirio y P. Mairal (Coords), Mayores Activos. Teorías, experiencias y reflexiones en torno a la participació social de las personas mayores (pp. 157-183). Madrid: La Factoría de Ediciones y Producciones.

2. Csikszentmihalyi, M. (1998). Creatividad: el fluir y la psicología del descubrimiento y la invención. Barcelona: Paidós.

3. Cummins, R. A. y Cahill, J. (2000). Avances en la comprensión de la calidad de vida subjetiva. Intervención Psicosocial, 9, 185-198.

4. Erlinghagen, M. y Hank, K. (2005). Participación de las personas mayores europeas en el trabajo de voluntariado. Boletín sobre el envejecimiento, 17, 1-24. Madrid: Ministerio de Trabajo y Asuntos Sociales-Secretaría de Estado de Servicios Sociales, Familias y Discapacidad.

5. Fernández-Ballesteros, R. y Maciá, A. (1993). Calidad de vida en la vejez. Intervención Psicosocial, 5, 77-94.

6. Herranz, I., Lirio, J. y Portal, E. (2012). Los mayores vistos por los profesores: Análisis cualitativo de las percepciones de los docentes. Actas del I Simposio Internacional Envejecimiento Activo y Solidaridad Intergeneracional: Claves para un Envejecimiento Activo. Madrid: UNED.

7. IMSERSO (2012). Informe 2010. Las personas mayores en España. Datos estadísticos nacionales. Observatorio de Personas Mayores. Madrid: IMSERSO-Ministerio de Sanidad, Política Social e Igualdad.

8. INE (2013). Cifras Oficiales de Población. Explotación estadística del padrón. Madrid: Instituto Nacional de Estadística.

9. INE (2011). Encuesta de Empleo del Tiempo 2009-2010. Madrid: Instituto Nacional de Estadística.

10. INE (2008). Encuesta Nacional de Salud 2006. Madrid: Instituto Nacional de Estadística y Ministerio de Sanidad, Política Social e Igualdad.

11. Lirio, J., Alonso, D. y Herranz, I. (2009). Envejecer Participando. El Proyecto "Entre Mayores". Una experiencia de investigación-acción. Buenos Aires: Miño y Dávila.
12. Marazza, E., Sarubbi, E., Castaldo, R., Chirre, A. y Denegri, D. (2003). La salud de adultos mayores en las representaciones de los jóvenes. Estudio en alumnos ingresantes de Psicología. En J. Sáez (Coord.), Educación y Aprendizaje en las personas mayores (pp. 253-268). Madrid: Dykinson.

13. Martínez, S., López, J.F., Amayra, I., Gómez, I. y Miguel, J.J. (2007). Participación y envejecimiento activo: Análisis de las percepciones de personas mayores implicadas en tareas de gestión en asociaciones de mayores. Revista Electrónica de Psicogerontología Tiempo, 21, 1-8. Recuperado el 14 de Enero de 2013 de (http://www.psiconet. com/tiempo).

14. Moen, P., Fields, V., Quick, H. E. y Hofmeister, H. (2000). A life-course approach to retirement and social integration. En: K. Pillemer y P. Moen (Eds.), Social integration in the second half of life (pp. 75-107). Baltimore, MD, US: The Johns Hopkins University Press.

15. Monchietti, A. y Krzemien, D. (2000). Participación social y estilo de vida. Su relación con la calidad de vida en la vejez. Revista Electrónica de Psicogerontología Tiempo, 21, 1-7. Recuperado el 14 de Enero de 2013 de (http:// www.psiconet.com/tiempo).

16. Omoto, A. M. y Snyder, M. (1995). Basic research in actino: volunteerins and society's response to AIDS. Personality and social psychology Bulletin 16, 152-165. http:// dx.doi.org/10.1177/0146167290161011

17. OMS. (2002). Envejecimiento activo, un marco político. Madrid: Organización Mundial de la Salud.

18. Pérez, M. (2002). La participación de las personas mayores. Apuntes para una agenda de intervenciones gerontológicas. Revista Interuniversitaria de Formación del Profesorado, 45, 21-32.

19. Yuni, J. A. y Urbano, C.A. (2007). La educación como factor de oportunidad para el desarrollo de las personas mayores. En D. Alonso, J. Lirio y P. Mairal (Coords.), Mayores Activos. Teorías, experiencias y reflexiones en torno a la participación social de las personas mayores. (pp. 87-117). Madrid: La Factoría de Ediciones y Producciones.

20. Vega, J. L., Buz, J. y Bueno, B. (2002). Niveles de actividad y participación social en las personas mayores de 60 años. Revista Interuniversitaria de Formación del Profesorado 45, 33-53.

Fecha de recepción: 7 de marzo de 2013 Fecha de recepción de la versión modificada: 27 de mayo de 2013 Fecha de aceptación: 18 de junio de 2013 